\title{
PANORAMA DE LOS OBSERVATORIOS DE POLÍTICAS PÚBLICAS EN IBEROAMÉRICA, 2000-2016
}

\author{
JOSÉ EDUARDO GUTIERREZ DURAN \\ Universidad Estatal a Distancia, Costa Rica \\ jgutierrezd@uned.ac.cr \\ JORGE ALBERTO VÁSQUEZ RODRÍGUEZ \\ Universidad Estatal a Distancia, Costa Rica \\ jvasquez@uned.ac.cr
}

\section{RESUMEN}

En Iberoamérica del siglo XXI han proliferado los observatorios de políticas públicas, surgidas en el Estado o en la Sociedad Civil como respuesta al reconocimiento general de la necesidad del estudio profundo de las políticas públicas que la nueva gobernanza y gobernabilidad exigen. Esto debido a que, hoy, la solución de los problemas públicos y la gestión pública requieren de la participación de todos los actores públicos y privados involucrados, que tienen características y relaciones cada vez más diversas y complejas, ya que en ellos se encuentra el conocimiento y los distintos recursos para el accionar efectivo del país.

PALABRAS CLAVE: POLÍTICAS PÚBLICAS, PROBLEMA PÚBLICO, PROBLEMA SOCIAL Y ESTADO.

\section{ABSTRACT}

In Latin America in the 21st century, public policy observatories have proliferated, arising in the State or in Civil Society, as a response to the general recognition of the need for in-depth study of the public policies that the new governance and governability demand, due to the that today the solution of public problems and public management, require the participation of all public and private actors involved, which have increasingly diverse and complex characteristics and relationships, since they contain knowledge and different resources for the effective action of the country.

KEYWORDS: PUBLIC POLICIES, PUBLIC PROBLEM, SOCIAL PROBLEM AND STATE. 


\section{INTRODUCCIÓN}

El objeto de este estudio son los 'Observatorios de políticas públicas' en Iberoamérica al año 2016; su identificación, naturaleza constitutiva y objetivos y fines. Es preciso clarificar ese objeto de estudio mediante la definición de los conceptos de política pública y observatorio, para luego construir un concepto orientador de 'Observatorio de políticas públicas', el que se utiliza en conjunto con aspectos teóricos de la gobernanza, la gobernabilidad y el neo-institucionalismo.

Seguidamente, en forma muy sucinta, se hará referencia a la metodología a través de la que se abordó el objeto de estudio, para exponer los hallazgos obtenidos en el proceso investigativo. Finalmente, se realizan las reflexiones finales, en especial, para el caso de Costa Rica.

\section{ASPECTOS TEÓRICOS}

Con el propósito de abordar el estudio de los observatorios de las políticas públicas, se estudiará, inicialmente, el concepto de 'políticas públicas.' Este está relacionado con el neoinstitucionalismo, fundamentado en actores y referido a la reivindicación y ampliación de la ciudadanía en el gobierno abierto (GA), en el marco de la nueva gobernanza y gobernabilidad, para luego construir el concepto de "observatorio de políticas públicas".

\section{POLÍTICA PÚBLICA}

La política pública, en su dimensión de bienes y servicios, es concebida como una respuesta a un problema social identificado como tal e incorporado al seno de discusión pública; esto último usualmente se le llama 'agenda política'. Cuando el problema social se incorpora en esa agenda política, se convierte en un problema público, pues el Estado interviene en la resolución del problema (Subirats et al., 2008; Vásquez, 2014).
En los años setenta y proveniente de las ciencias políticas, especialmente en los Estados Unidos de América, se introduce el término 'política pública', cuyo objeto principal es la solución de los problemas públicos, reconocidos como tales. Estos se refieren a las interacciones, alianzas y conflictos, en un marco institucional específico, entre los diferentes actores públicos, parapúblicos y privados, para resolver un problema público que requiere de una acción concreta (Subirats et al., 2008, Vásquez, 2014).

Según Subirats et al. (2008), se requiere de una definición 'operacional' para calificar el objeto y el campo de estudio de la disciplina. Desde esa perspectiva, debe entenderse política pública como

... una serie de decisiones o de acciones, intencionalmente coherentes, tomadas por diferentes actores, públicos y a veces no públicos -cuyos recursos, nexos institucionales e intereses varían- a fin de resolver de manera puntual un problema políticamente definido como colectivo. Este conjunto de decisiones y acciones da lugar a actos formales, con un grado de obligatoriedad variable, tendentes a modificar la conducta de grupos sociales que, se supone, originan el problema colectivo a resolver (grupos-objetivos), en el interés de grupos sociales que padecen los efectos negativos del problema en cuestión (beneficiarios finales) (Subirats et al., 2008, p. 38).

De la anterior definición, surge la importancia del actor y de las instituciones que se tratan a continuación.

\section{NEOCONSTITUCIONALISMO}

De la definición de la política pública se evidencia que se fundamenta en la corriente teórica del neoinstitucionalismo, basada en actores. Existen dos elementos clave en esa conceptualización: 
las reglas institucionales y los actores, los cuales se influyen mutuamente. Esta concepción se aleja del institucionalismo en el hecho de que las reglas institucionales determinan las decisiones individuales y colectivas de los actores.

Después de 1980 se dio un viraje en el vínculo entre instituciones y actores, y se considera que existe una relación recíproca entre ambos. En otras palabras, se parte de que las instituciones no determinan ni limitan, en forma absoluta, las actuaciones de los actores. A partir de aquí, surge lo que se conoce como 'neoinstitucionalismo'.

\section{El neoinstitucionalismo}

... postula que los actores y las instituciones se influyen recíprocamente. Según este paradigma, el homo institutionalus', al encontrarse 'incrustado 'o 'atrapado' en las reglas institucionales formales e informales, adopta conductas políticas que se adecuan a los valores y a las expectativas que las citadas reglas proponen, pero al mismo tiempo las modifica de manera incremental mediante sus propias decisiones y acciones (Subirats et al., 2008, p. 211).

Un importante y contundente estudio del institucionalismo centrado en los actores es el de Scharpf (1997), en el que se destaca la incidencia que las instituciones

... tienen sobre las percepciones, las preferencias y las capacidades de actores individuales o colectivos $y$, por otro, sobre las formas en que estos actores interactúan. Las instituciones son, sin duda, la principal fuente de información de los actores y el principal factor que influye sobre sus decisiones [... las instituciones no influyen en las deci-

Se interpreta que el homo institutionalus es el hombre institucionalizado, es decir, el hombre cuya conducta se encuentra en función de la instituciones formales e informales (Subirats et al., 2008, p. 211). siones de una manera determinista, puesto que siempre existen distintas posibilidades de acción que dejan un amplio margen para que los actores elijan entre diferentes opciones tácticas y estratégicas (Scharpf, 1997, citado por Zurbriggen, 2008, p. 6).

En esa concepción de Scharpf, se reconoce que los resultados de una política pública no son solo el de las reglas institucionales, sino la actuación de los actores que se rigen en ese marco institucional. Cada actor está institucionalizado en tanto surge de una arena política con reglas determinadas, las que permiten su incursión, permanencia y expulsión de esa arena política, de ahí que las instituciones operan como facilitadoras pero, también, establecen la actuación del actor (Vásquez, 2017). En el modelo de Sharpf, 1997, los

... actores se caracterizan por sus recursos o capacidades, que les proporcionan mayor o menor influencia en el resultado final, y también por sus percepciones de la realidad y sus preferencias, las cuales pueden ser más o menos estables, pero también sujetas a cambios en los que, como se ha visto, influye el contexto institucional (Zurbriggen, 2008, p. 7).

El enfoque teórico de Sharpf es, entonces, neoinstitucional en tanto el contexto institucional no tiene una influencia absoluta sobre el actor. La constelación de actores es un aspecto esencial en el modelo de Sharpf, ya que en él no existe un actor absoluto que determina la política pública, sino una serie de actores que participan en ella, a los que se le debe brindar atención a

... sus posibles estrategias de acción con respecto a ella, a los posibles resultados que tendrían lugar a partir de las diferentes estrategias de acción de los actores y, por último, a las preferencias o valoración de los 
actores con respecto a tales resultados posibles (Zurbriggen, 2008, p. 7).

Así pues, si bien la influencia del actor es importante, lo es más la actuación de un conjunto de actores que pueden determinar el rumbo de una política pública específica. El enfoque de Sharpf, 1997, citado por Zurbriggen, 2008, establece un tercer elemento al que denomina 'modos de interacción', que son

... las formas en que unos actores se conducen con respecto a los otros, los cuales están en gran medida condicionados por el contexto institucional en el que se desenvuelven. Cada modo de interacción está regulado por un sistema de pautas institucionales para el uso de ese modo en cuestión. Pero, además, está influenciado por un sistema institucional más amplio, dentro del cual tienen lugar las interacciones (Zurbriggen, 2008, p. 7).
La política pública debe analizarse y entenderse con base en el conjunto de la constelación de actores. Rara vez estos, o sus modos de interacción, son definidos/clasificados en un marco institucional específico. En ese marco, se ayuda a fijar tanto la constelación de actores como la interacción entre ellos. La Figura 1 ilustra el institucionalismo centrado en actores.

\section{CLASIFICACIÓN DE LOS ACTORES}

El actor es un componente fundamental en la política pública, al igual que lo es el institucionalismo. De esta manera, por medio de actores se formula e implementa la política y su destinatario son también actores.

Todo individuo, persona jurídica o grupo social se considera un actor desde el momento en que, por el solo hecho de existir, pertenece a un campo social considerado como pertinente

FIGURA 1

NEOINSTITUCIONALISMOS CENTRADO EN ACTORES, MARCO EXPLICATIVO BÁSICO

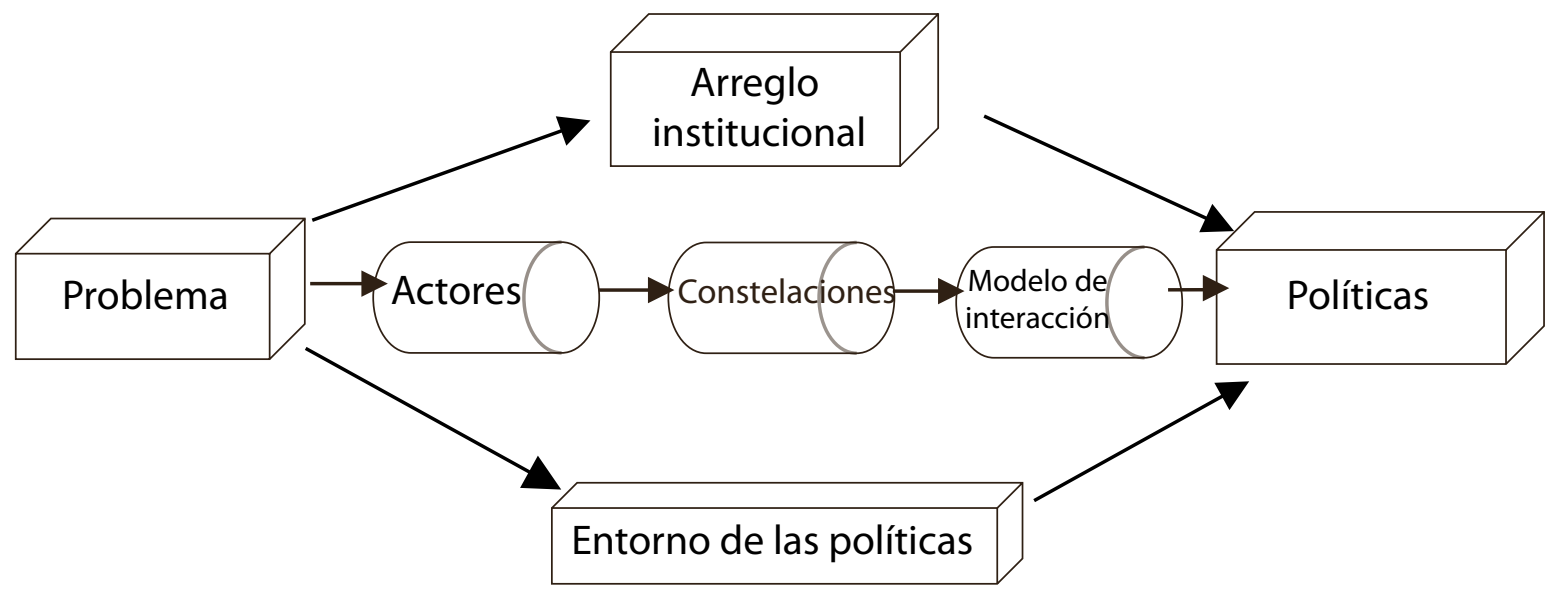

Fuente: Sharpf, 1997, citado por Zurbriggen, 2008 
para el análisis" (Subirats et al., 2008, p. 52), además del que obtiene esa condición por

... el simple hecho de formar parte del campo estudiado, en la medida que el análisis de su comportamiento contribuye a estructurar el campo. No es pues un problema de conciencia, de lucidez o de identificación: es una simple cuestión de hecho, lo que implica que acaba siendo una cuestión de investigación (Friedberg, 1993, p. 199).

De conformidad con Subirats et al. (2006), los actores de las políticas públicas son los siguientes:

i. Las autoridades políticas administrativas, que a su vez se dividen en actores públicos y actores paraestatales.

ii. Los actores privados, que a su vez se dividen en grupos-objetivo, beneficiarios finales y grupos terceros. Los primeros se identifican usualmente como los causantes del problema público que se pretende solucionar. Los otros son los beneficiados de esa política pública.

\section{CIUDADANO Y POLÍTICAS PÚBLICAS}

Todo individuo o grupo social implicado en cualquiera de las fases de formulación, implementación y evaluación de una política pública y sus beneficiarios se consideran un actor de una política pública. En el siglo XXI existe un mayor acercamiento del Gobierno al ciudadano y este ha visto reivindicados y ampliados sus derechos ciudadanos, lo que lo hace un actor de mayor relevancia (Breppe y Pereyra, 2014).

Actualmente, ya no se habla de un ciudadano pasivo del siglo XX, cuya participación en la democracia representativa se circunscribía a una votación que realizaba cada periodo para elegir al representante del Poder Ejecutivo y del Poder Legislativo o a su gobierno local
(Breppe y Pereyra, 2014). Por el contrario, el ciudadano amplía su ámbito de acción y se convierte en un fiscalizador de los actos públicos, de los funcionarios elegidos directamente por él o de aquellos que conforman el aparato burocrático del Estado. Además, exige más y mejores servicios, propone proyectos y colabora con su ejecución.

El gobierno abierto (GA) en el presente siglo es el

... que abre sus puertas al mundo, co-innova con todos, especialmente con los ciudadanos; comparte recursos que anteriormente estaban celosamente guardados, y aprovecha el poder de la colaboración masiva, la transparencia en todas sus operaciones, y no se comporta como un departamento o una jurisdicción aislada, sino como una estructura nueva, como una organización verdaderamente integrada y que trabaja en red (Lathrop y Ruma, 2010, p. XVI).

En los últimos años, la idea del GA

... incluye la necesidad de contar con gobiernos "transparentes", que provean nuevas avenidas para dar más acceso a la información pública, y que "rindan cuentas" para incentivar el control social de la gestión pública y la prevención de la corrupción. Pero ello no se agota allí. También las políticas públicas de transparencia se orientan a la producción proactiva de información (y datos públicos abiertos) a fin de brindar una mejor calidad en el servicio público y promover una mayor utilización de dichos insumos por parte de los ciudadanos/beneficiarios. Por ello, no se trata únicamente de publicar más información, sino de que esta sea de mejor calidad, útil y focalizada, para que los usuarios actuales y potenciales de los servicios públicos tomen mejores decisiones, y que sea publicada de determinados modos y en determinados forma- 
tos para hacerla más inteligente (Ramírez y Dassen, 2012, p. 43)

En el gobierno abierto (GA) los ciudadanos participan en el monitoreo o fiscalización de las políticas públicas, además de contribuir ampliamente en ellas, tanto como elaboradores, coejecutores o fiscalizadores.

\section{LA POSICIÓN TEÓRICA}

De conformidad con las teorías antes expuestas sobre política pública, neonstitucionalismo y actores, se toma una posición teórica que se expone a continuación. En primer lugar, no todo problema social es un problema público, aunque todo problema público es un problema social. Se diferencian en que este último es candidato a ser considerado parte de la agenda política; en cambio, el problema público ya se encuentra en dicha agenda. La política pública desde el Estado, con participación de múltiples actores, incluso privados, pretende brindar una solución a un problema público reconocido como tal.

En la formulación de la política pública, en la cual es importante la hipótesis de solución a las causas del problema público, concurren múltiples actores públicos y privados; se establece, generalmente, mediante una normativa. En el caso de Costa Rica, es una ley o un reglamento del Poder Ejecutivo; en ella, se establecen las reglas formales, es decir, las denominadas "reglas del juego", así como los actores que participan en ellas De esta forma, se les otorga, reconoce y se hace evidente los recursos con que cuentan esos actores.

La política pública coexiste con otras. Se debe considerar la interrelación de esas políticas y debe considerar e incluso subordinarse cualquier propuesta a una serie de normas formales (algunas de rango superior). Para el caso de Costa Rica, sería la Constitución Política y los trata- dos internacionales y otras transversales, que a su vez cuentan con sus actores y recursos.

En la interrelación de instituciones y de actores de una política, surgen también una serie de reglas informales que influyen en la política, y por tanto, es básico considerarlas. Las reglas formales y los actores se influyen mutuamente; ningún actor es preponderante por sí mismo, sino lo es la red de actores, la cual podría estar liderada por uno o más actores.

Una política pública es, entonces, una serie de decisiones o de acciones intencionalmente coherentes tomadas por diferentes actores, públicos y, a veces, no públicos, cuyos recursos, nexos institucionales e intereses varían a fin de resolver de manera puntual un problema políticamente definido como público. Debido a la reivindicación y a la ampliación de los derechos ciudadanos, el ciudadano no es un actor pasivo y receptivo de los bienes y servicios producidos por las políticas públicas, sino que monitorea fiscaliza, participativa y hasta coadyuva en su formulación e implementación.

Con el gobierno abierto, el ciudadano es partícipe en la formulación e implementación de las políticas públicas, además de ser su acérrimo vigilante. Una vez dilucidado el concepto de política pública, hay que adentrarse en la noción de 'observatorio'.

\section{OBSERVATORIO}

Los observatorios de fenómenos sociales tienen su origen en los observatorios astronómicos. Aunque la observación de los astros se remonta a miles de años atrás, los primeros observatorios astronómicos se localizan alrededor del año 1000 a. C. con la 'torre o zigurat de Belo' en Babilonia y los astrónomos caldeos.

Los observatorios fueron perfeccionados en el siglo VIII a. C.; otros, fueron creados en Europa, como el de la Giralda de Sevilla, a finales de la 
Edad Media; el Landgrave de Hesse-Cassel, en 1561; el de Tycho Brahe, en 1576. En el siglo $X X I$, se registraron cientos de ellos alrededor del mundo. El observatorio astronómico es una institución desde la cual se investiga y se registran objetos, eventos y situaciones relacionados con fenómenos astronómicos, es un observador permanente del espacio.

El concepto de 'observatorio' fue trasladado de las ciencias astronómicas al ámbito social, con un fin similar al de dicho ámbito: la investigación y registro de eventos y situaciones sociales. En el ámbito social, en el siglo XXI, existe una proliferación de observatorios para el abordaje de asuntos muy distintos. Estos se fundaron en organismos internacionales y en gobiernos de los diferentes países, desde el Estado, la sociedad civil o de asociaciones públicas y privadas.

Desde la sociedad civil, se formaron los denominados 'observatorios ciudadanos'. Por lo general, se trata de asociaciones sin fines de lucro y dependen del financiamiento de agencias internacionales, estados nacionales o contribuciones de empresas o ciudadanos (Natal y Díaz, 2014). Los observatorios de los asuntos sociales rescatan una parte fundamental de la naturaleza de sus homólogos astronómicos, por su labor permanente y continua respecto al asunto social para el que fueron creados.

El Instituto Nacional de Administración Pública define observatorio como

... un organismo creado por un colectivo con el fin de seguir la evolución de un fenómeno de interés general; desde las administraciones públicas, nacionales, regionales y locales; desde instituciones académicas, sindicatos, empresas, fundaciones, organizaciones de la sociedad civil se ha promovido y apoyado la creación de estos órganos coadyuvantes al desempeño institucional mediante señalamientos puntuales y propositivos previo estudio, registro y análisis de la situación y evolución de una temática determinada (2014, p. 15).

En la presente investigación, se entiende como observatorio de políticas públicas aquel organismo creado desde los organismos internacionales, las instituciones de un Estado, las empresas u organizaciones de la sociedad civil o en alianzas público-privadas que se creó con el fin de seguir la evolución de las políticas públicas.

Los actores son importantes porque, junto con las instituciones, son elementos de relevancia en el estudio de las políticas públicas. Asimismo, dichos actores son los que fundan, gestionan, operan y participan en esos observatorios y, por ello, sus intereses están impregnados en la cotidianidad de los observatorios.

\section{NUEVA GOBERNANZA Y GOBERNABILIDAD}

La creación de los observatorios se encuentra asociada a la nueva gobernanza y la gobernabilidad. Desde la década de 1990 se utiliza el concepto de gobernanza, para hacer referencia a la eficacia, calidad y buena orientación de la intervención del Estado. Seguidamente, surgió el término de gobernabilidad para referirse al proceso mediante el cual diversos actores, tanto públicos como privados, ejercen el poder y la autoridad y, al hacerlo, toman e implementan decisiones en el ámbito público (Mayorga y Córdoba, 2007; Breppe y Pereyra, 2014).

La nueva gobernanza y gobernabilidad exigen de las autoridades públicas tomar en cuenta a los diversos actores públicos y privados en la formulación e implementación de las políticas públicas. A su vez, las autoridades deben estar legitimadas democráticamente. La participación ciudadana se ha convertido en un factor que no es obligatorio en términos absolutos pero sí necesario para llevar a cabo acciones públicas de una forma eficiente y eficaz, en democracia (Mayorga y Córdoba, 2007). 
Entonces, la gobernabilidad es la capacidad para crear las condiciones óptimas para la formulación e implementación de políticas públicas y de la gestión pública en general. También, se le considera en la nueva gobernanza para lograr la eficacia, eficiencia y una elevada calidad de la gestión pública. La nueva ciudadanía, con niveles educativos formales e informales cada vez mayores, con reconocimiento de sus derechos, en un mundo interconectado e interdependiente, cada vez con mayor participación y conocimiento de la gestión pública en función de la gestión privada, hace necesario un conocimiento mayor de las políticas públicas, que son el objeto de estudio de los observatorios de políticas públicas.

\section{METODOLOGÍA}

Se realizaron consultas documentales de las universidades públicas de Costa Rica, especialmente. Fueron revisadas las investigaciones del Doctorado en Gobierno y Políticas Públicas de la Universidad de Costa Rica. Se consultaron los sitios web de los observatorios de políticas públicas de Iberoamérica, así como una serie de investigaciones realizadas por la Comisión Económica para América Latina (Cepal).

La información recopilada sobre los observatorios de Iberoamérica fue objeto de análisis a partir de cuadro sinóptico, con el propósito de verificar coincidencias y divergencias. A través del análisis del discurso, se descubre, en parte, los intereses de los actores que operan los observatorios; especialmente, se estudia mediante dicho método la misión, la visión y los objetivos externados o asociados a los observatorios.

\section{LOS OBSERVATORIOS DE POLÍTICAS PÚBLICAS EN IBEROAMÉRICA}

La creación de observatorios de políticas públicas en Iberoamérica está en auge desde el año 2002. Se promueve su apertura desde el
Estado, de la sociedad civil y hasta del mercado e incluso impulsados por organismos internacionales. Lo anterior surge como respuesta a los requerimientos de la nueva gobernanza y gobernabilidad, en tanto los ciudadanos en Iberoamérica, son más conscientes de sus derechos y necesidades y se encuentran en un mundo interconectado, lo que hace que se encuentren informados.

Los gobernantes en general y los activistas de la Sociedad Civil requieren un mayor conocimiento de la interacción de los actores públicos y privados que intervienen en la formulación e implementación de las políticas públicas. Por ello, surgen los observatorios como una respuesta en Iberoamérica a esa necesidad de conocimiento de la ciudadanía en su interacción en lo público. Esas necesidades de conocimiento de las políticas públicas llevan a la fundación de una serie de observatorios, prácticamente en todos los países de lberoamérica.

Muchos de los observatorios en Iberoamérica se dedican al estudio de las políticas públicas generales; otros, a políticas públicas particulares. Unos surgen en el Estado y otros en la Sociedad Civil. Unos son de carácter nacional y otros de carácter internacional. Podrían clasificarse de dos formas:

i) Naturaleza jurídica de la organización: público internacional, público nacional, público local, privado, académicas y alianzas privadas o público-privadas.

ii) Materia.

Como puede apreciarse, en los últimos años los observatorios fueron creados en el marco de la transformación de las administraciones públicas, de cara al post-Consenso de Washington. En ellos prevalece el imperioso requerimiento del "Estado necesario", en el que este converge con el mercado y la Sociedad Civil en tamaño, forma y relaciones propias de cada país. 


\section{OBSERVATORIOS DE POLÍTICAS PÚBLICAS DE NATURALEZA PÚBLICO INTERNACIONAL}

Existe una serie de observatorios de políticas públicas que operan desde organismos internacionales o son impulsados por ellos. En Latinoamérica, la Comisión Económica para América Latina (Cepal) ha participado en la fundación o co-fundación de los siguientes observatorios en distintos ámbitos: Observatorio para la Sociedad de la Información en Latinoamérica y el Caribe, en el 2003; Observatorio de Igualdad de Género de América Latina y el Caribe en el 2007; Observatorio de Juventud para América Latina y el Caribe; Observatorio del Principio 10 de América Latina y el Caribe; Observatorio América Latina-Asia Pacifico; Observatorio Fiscal de Latinoamérica y el Caribe; Observatorio Regional de Banda Ancha en el 2010.

La Cepal, de conformidad con su naturaleza de organización regional, impulsa el estudio de las políticas públicas en los diferentes países de Latinoamérica con la creación de los observatorios. No obstante, considera una política pública concreta los temas sobre la igualdad de género, el aspecto fiscal o la información.

Ejemplo de ese interés regional de la Cepal es el Observatorio de Igualdad de Género de América Latina y el Caribe, a cargo de dicha comisión a través de la División de Asuntos de Género. Este observatorio monitorea las políticas públicas en todos los países miembros de la Cepal, relacionadas con asuntos de género; por lo tanto, es un generador de estudios y de indicadores relacionados con esta materia.

De igual forma que la Cepal, la Organización de las Naciones Unidas para la Educación, la Ciencia y la Cultura (Unesco) impulsa una serie de observatorios en el mundo con los temas de la ciencia y la cultura, por ejemplo: el Observatorio sobre Movilidades Académicas y Científicas en América Latina y el Caribe; el Observatorio de Diversidad Cultural e Interculturalidad; el Observatorio de Responsabilidad Social para América Latina y el Caribe y el Observatorio Pensamiento Universitario Latinoamericano.

De esta forma, el Observatorio de Diversidad Cultural e Interculturalidad en Educación Superior, impulsado por la Unesco

... procura contribuir con el avance, profundización y crecimiento de las experiencias de educación superior intercultural actualmente existentes en América Latina y el Caribe, así como con los debates y reformas tendientes a lograr que toda la educación superior sea pertinente con la diversidad cultural propia de cada país de la región (Unesco, 2017).

\section{OBSERVATORIOS DE POLÍTICAS PÚBLICAS DE NATURALEZA PÚBLICO NACIONAL}

Desde la primera década del siglo XXI, varias organizaciones públicas de distintos países en Iberoamérica formaron observatorios de políticas públicas con el propósito fundamental de coadyuvar con la formulación e implementación de las políticas, sistematizar las experiencias y capacitar a los funcionarios públicos. Así, en Argentina se fundaba el Observatorio de Políticas Públicas del Cuerpo de Administradores Gubernamentales y el Observatorio Argentino de Drogas; en Brasil, el Observatorio Brasileño de la Economía Creativa y el Observatorio de Política Nacional del Control de Tabaco.

Además, en Bolivia surgió el Observatorio Bolivia Autonómica; en Chile, el Observatorio Habitacional Ministerio de Vivienda y Urbanismo y el Observatorio Chilecompra; en Colombia, el Observatorio de Políticas Públicas de Medellín y el Observatorio de Políticas Públicas de Medellín; en Ecuador, el Sistema Nacional de Información y el Observatorio de Seguridad Ciudadana del Distrito Metropolitano de Quito. 
También, en México aparece el Observatorio de Violencia Social y de Género de México; en Paraguay, el Observatorio de Recursos Humanos en Salud y el Observatorio Laboral; en Ecuador, el Observatorio de Seguridad Ciudadana del Distrito Metropolitano de Quito; en Uruguay, el Observatorio Uruguay de Políticas Públicas y, en Venezuela, el Observatorio Venezolano de Seguridad Ciudadana. La fecha de creación de cada observatorio, así como su fecha de creación por país, se puede observar en la Tabla 1.

La gran cantidad de observatorios de políticas públicas creada en los diferentes países es una muestra de la conciencia que han tomado los gobernantes de Iberoamérica de estudiar para su mejoramiento las distintas políticas públicas, con el objetivo de producir bienes y servicios destinados a una ciudadanía cada vez más exigente. Esta conciencia de los gobernantes proviene del reconocimiento de que la eficacia y la eficiencia de una política pública no dependerán únicamente de la gestión del gobernante per se, sino de la consideración y asocio de otros actores, provenientes del mismo Estado y de la sociedad civil. Todo esto en un marco de reivindicación y de ampliación de los derechos ciudadanos dentro de un proceso de mejoramiento de la democracia representativa.

Estos observatorios son creados como un departamento o una dependencia más que un órgano del Estado. Por esta razón, los esfuerzos del observatorio se encuentran en concordancia con los planes, programas o decisiones de las autoridades públicas, de ahí que no exista una cierta "autonomía" con respeto a los actores públicos, que amarre la capacidad crítica de los investigadores en políticas públicas.

\section{OBSERVATORIOS DE POLÍTICAS PÚBLICAS DE NATURALEZA PÚBLICO LOCAL}

Existen observatorios de políticas públicas de gobiernos locales. En Colombia desde el año
2002, opera el Observatorio de Políticas Públicas de Medellín y se encuentran aprobados, por parte de sus gobiernos locales, observatorios en políticas públicas para los distritos de Barranquilla y en Cartagena de Indias. En México, el Gobierno de Coahuila tiene el Observatorio de Políticas Públicas para la Innovación Gubernamental en Coahuila, sus dependencias gubernamentales y municipios.

Existen otros observatorios de políticas públicas de gobiernos locales, pero de una política pública específica. Por ejemplo, en México existen el Observatorio Ciudadano de Seguridad Pública del Municipio de Puebla, el Observatorio Ciudadano de Seguridad y Convivencia de la Ciudad de Oaxaca el Observatorio Ciudadano de Transparencia y Gestión Municipal del Municipio de Aguascalientes.

Esos observatorios son más focalizados. Se relacionan con colaboración de la formulación e implementación de políticas públicas en una determinada fracción de un territorio nacional. También, tienen distinta denominación según cada país: provincia, distrito, cantón o departamento, según investigaciones y capacitaciones en el campo de las políticas públicas.

Al igual que en los observatorios de políticas públicas de naturaleza público nacional, en los observatorios de políticas públicas de naturaleza público local se observa el reconocimiento de las autoridades públicas que la eficiencia y la eficacia de una política pública local dependerá de la participación de un grupo de actores. Unos de ellos son públicos en el ámbito nacional; otros, actores públicos locales y actores provenientes del ámbito privado, desde la sociedad civil y del mercado. Los objetivos de estos observatorios se encuentran en armonía con los objetivos de las organizaciones públicas locales que integran. 
TABLA 1

IBEROAMÉRICA: OBSERVATORIOS DE POLÍTICAS PÚBLICAS DEL ESTADO

\begin{tabular}{|c|c|c|c|}
\hline País & Observatorio & Creado & Sitio web \\
\hline \multirow[t]{3}{*}{ Argentina } & $\begin{array}{l}\text { Observatorio de Políticas Públicas del Cuerpo de } \\
\text { Administradores Gubernamentales }\end{array}$ & 2002 & $\begin{array}{l}\text { http://www.sgp.gov.ar/contenidos/ag/paginas/opp/opp. } \\
\qquad \text { html }\end{array}$ \\
\hline & Observatorio Argentino de Drogas & 2002 & $\begin{array}{l}\text { http://www.oas.org/es/sap/dgpe/pub/observatoriosde- } \\
\text { politicaspublicas_s.pdf }\end{array}$ \\
\hline & Observatorio de Políticas Públicas y Desarrollo & 2005 & $\begin{array}{l}\text { http://flacso.org.ar/investigaciones/observatorio-en-poli- } \\
\text { ticas-publicas-y-desarrollo/ }\end{array}$ \\
\hline \multirow[t]{2}{*}{ Brasil } & Observatorio Brasileño de la Economía Creativa & 2012 & http://www2.cultura.gov.br/economiacriativa/ \\
\hline & Observatorio de Política Nacional del Control de Tabaco & 2002 & http://www.observatorio.gov.ar/queeseload.html \\
\hline Bolivia & Observatorio Bolivia Autonómica & & $\begin{array}{l}\text { https://www.facebook.com/ObservatorioBoliviaAutono- } \\
\text { mica/ }\end{array}$ \\
\hline \multirow[t]{2}{*}{ Chile } & Observatorio Chilecompra & 2013 & $\begin{array}{l}\text { http://observatorio.chilecompra.cl/index.php?option=- } \\
\text { com_content\&view=article\&id=104\&ltemid=506 }\end{array}$ \\
\hline & Observatorio Habitacional Ministerio de Vivienda y Urbanismo & No hay dato & $\begin{array}{l}\text { http://www.observatoriohabitacional.cl/opensi- } \\
\text { te_20080122170754.aspx }\end{array}$ \\
\hline Colombia & Observatorio de Políticas Públicas de Medellín & 2002 & $\begin{array}{l}\text { https://www.medellin.gov.co/irj/portal/medellin?Naviga- } \\
\text { tionTarget=navurl://02ea3f8dac7a75cba98039fe75b7bef1 }\end{array}$ \\
\hline \multirow[t]{3}{*}{ España } & Observatorio de la Violencia de Género & 2005 & http://observatorioviolencia.org/ \\
\hline & Observatorio de Contratación Pública & 2011 & http://www.obcp.es/ \\
\hline & Observatorio de Políticas de Agua & 2013 & https://fnca.eu/oppa \\
\hline \multirow[t]{2}{*}{ Ecuador } & Sistema Nacional de Información & 2009 & $\begin{array}{l}\text { http://www.oas.org/es/sap/dgpe/pub/observatoriosde- } \\
\text { politicaspublicas_s.pdf }\end{array}$ \\
\hline & $\begin{array}{l}\text { Observatorio de Seguridad Ciudadana del Distrito } \\
\text { Metropolitano de Quito }\end{array}$ & 2003 & $\begin{array}{l}\text { http://www.oas.org/es/sap/dgpe/pub/observatoriosde- } \\
\text { politicaspublicas_s.pdf }\end{array}$ \\
\hline México & Observatorio de Violencia Social y de Género de México & 2009 & http://www.gob.mx/indesol/que-hacemos \\
\hline \multirow[t]{2}{*}{ Paraguay } & Observatorio de Recursos Humanos en Salud & 2009 & http://observatorioparaguayorhus.ning.com/ \\
\hline & Observatorio Laboral & No hay dato & http://www.sinafocal.gov.py/index.php?cID=272 \\
\hline Perú & Observatorio Peruano de Drogas & No hay dato & $\begin{array}{l}\text { http://www.devida.gob.pe/institucion/direc- } \\
\text { cion-de-asuntos-tecnicos/opd/ }\end{array}$ \\
\hline Uruguay & Observatorio Uruguay de Políticas Públicas & 2013 & $\begin{array}{c}\text { http://www.agev.opp.gub.uy/observatorio/servlet/ } \\
\text { maininicio }\end{array}$ \\
\hline Venezuela & Observatorio Venezolano de Seguridad Ciudadana & 2012 & http://www.ovs.gob.ve/index.php?lang=en \\
\hline
\end{tabular}

Fuente: Gutiérrez y Vásquez (2017) 


\section{OBSERVATORIOS DE POLÍTICAS PÚBLICAS DE NATURALEZA PRIVADA}

En Iberoamérica se pueden identificar observatorios provenientes de organizaciones privadas: unas con fines de lucro y otras denominadas organizaciones no gubernamentales, pero principalmente de estas últimas. En Brasil, se forman observatorios de políticas públicas denominados 'observatorio social.' Entre ellos, se encuentran el Observatorio Social de Brasil, fundado en el 2008 y el Observatorio de San José, establecido en el 2011. Estos tienen como fin el monitoreo o la colaboración con la formulación e implementación de las políticas públicas. Los ciudadanos de la gestión pública son fiscalizadores y fomentan la transparencia de las actuaciones públicas.
En República Dominicana, existen el Observatorio Político Dominicano y el Observatorio Dominicano de Políticas Públicas, que realizan estudios sobre políticas públicas y colaboran con capacitación de funcionarios públicos. En la tabla 2, se puede observar una serie de observatorios que nacen desde el ámbito privado, especialmente, desde las organizaciones no gubernamentales.

Del estudio de esas organizaciones, se puede extraer que tienen un mayor énfasis en la fiscalización de la gestión pública, en la utilización de los recursos públicos y en los procesos de transferencias. Al formar parte de la sociedad civil, no dependen necesariamente de la ayuda Estatal y no deben seguir lineamientos estatales, aunque

TABLA 2

IBEROAMÉRICA: OBSERVATORIOS DE POLÍTICAS PÚBLICAS DE NATURALEZA PRIVADA, 2016

\begin{tabular}{|c|c|c|c|}
\hline País & Observatorio & Creado & Sitio web \\
\hline \multirow[t]{2}{*}{ Argentina } & Observatorio Pyme & 2005 & http://www.observatoriopyme.org.ar/ \\
\hline & Observatorio de Políticas Públicas y Desarrollo & 2005 & $\begin{array}{l}\text { http://flacso.org.ar/investigaciones/observatorio-en-politi- } \\
\text { cas-publicas-y-desarrollo/ }\end{array}$ \\
\hline \multirow[t]{2}{*}{ Brasil } & Observatorio Social de Brasil & 2008 & http://osbrasil.org.br/o-que-e-um-observatorio-social-os/ \\
\hline & Observatorio Social de San José & 2011 & http://www.ossj.org.br/novo/quem-somos/ \\
\hline Bolivia & Observatorio de Género de la Coordinadora de la Mujer & $2010^{2}$ & $\begin{array}{l}\text { http://www.coordinadoradelamujer.org.bo/observatorio/index. } \\
\text { php/qsomos/menu }\end{array}$ \\
\hline \multirow[t]{3}{*}{ España } & Observatorio de la Violencia de Género & 2005 & http://observatorioviolencia.org/ \\
\hline & Observatorio de Contratación Pública & 2011 & http://www.obcp.es/ \\
\hline & Observatorio de Políticas de Agua & 2013 & https://fnca.eu/oppa \\
\hline \multirow[t]{2}{*}{ México } & Observatorio de Familias y Políticas Públicas & 2003 & $\begin{array}{l}\text { http://www.incidesocial.org/index.php?option=com_conten- } \\
\text { t\&task=view\&id=31\&ltemid=37 }\end{array}$ \\
\hline & Observatorio de Políticas Públicas y de Salud de México & 2015 & $\begin{array}{l}\text { http://conacytprensa.mx/index.php/ciencia/salud/11997-ob- } \\
\text { servatorio-de-politica-publica-y-de-salud-de-mexico }\end{array}$ \\
\hline Portugal & Observatorio político & 2009 & $\begin{array}{c}\text { http://www.iscsp.ulisboa.pt/index.php?option=com_conten- } \\
\text { t\&view=article\&id=2037\&ltemid }=470\end{array}$ \\
\hline \multirow{2}{*}{$\begin{array}{l}\text { República } \\
\text { Dominicana }\end{array}$} & Observatorio Político Dominicano & $2010^{2}$ & http://www.opd.org.do/ \\
\hline & $\begin{array}{c}\text { Observatorio Dominicano de Políticas Públicas e } \\
\text { investigación }\end{array}$ & 2011 & http://odppuasd.blogspot.com/ \\
\hline
\end{tabular}

Fuente: Gutiérrez y Vásquez (2017)

Algunas fuentes señalan otro año de creación. 
sus fines sean de naturaleza pública, por lo que les brinda una mayor independencia de criterio.

\section{OBSERVATORIOS DE POLÍTICAS PÚBLICAS DE NATURALEZA ACADÉMICA}

De las universidades en Iberoamérica surgen una serie de observatorios de políticas públicas. Se dedican, principalmente, a la investigación y a la producción de índices sobre algunas políticas públicas específicas.

Estos observatorios tienen un énfasis en la investigación de temas específicos. El caso del Observatorio de Políticas Públicas y Desarrollo de Argentina, por ejemplo, tiene como objetivo promover y contribuir al desarrollo de investiga- ciones académicas y soluciones prácticas, vinculados al Modelo de Desarrollo, el Estado y a las políticas públicas en Argentina y en la región latinoamericana. Los objetivos específicos de dicho observatorio se relacionan con la compilación, la producción, el análisis y la comunicación de las investigaciones relacionadas con las políticas públicas de Argentina y de Latinoamérica.

Los observatorios de políticas públicas de naturaleza académica tienen su propia agenda, que no necesariamente es coincidente con la de las autoridades públicas. En consecuencia, podrían estar realizando investigaciones que, desde la perspectiva académica, son interesantes pero, desde el criterio de los gobernantes, no lo son.

TABLA 3

IBEROAMÉRICA: OBSERVATORIOS DE POLÍTICAS PÚBLICAS ASOCIADOS A UNIVERSIDADES EN IBEROAMÉRICA

\begin{tabular}{|c|c|c|}
\hline País & Observatorio & Sitio web \\
\hline Argentina & Observatorio de políticas públicas y desarrollo & $\begin{array}{l}\text { http://flacso.org.ar/investigaciones/observatorio-en-politi- } \\
\text { cas-publicas-y-desarrollo/ }\end{array}$ \\
\hline Brasil & Observatorio de Política Nacional del Control del Tabaco & \\
\hline \multirow[t]{5}{*}{ Costa Rica } & $\begin{array}{l}\text { Observatorio Laboral de Profesiones del Consejo } \\
\text { Nacional de Rectores }\end{array}$ & http://olap.conare.ac.cr/ \\
\hline & Observatorio de Política Internacional & http://opi.ucr.ac.cr/ \\
\hline & Observatorio Ambiental & http://www.observatorioambiental.una.ac.cr/ \\
\hline & Observatorio de Mipymes & $\begin{array}{l}\text { https://www.innovacion.cr/blog/observatorio-de-mipymes } \\
\text { www.omipymes.uned.ac }\end{array}$ \\
\hline & Observatorio de la Educación Nacional y Regional & http://observatorio.inie.ucr.ac.cr/ \\
\hline \multirow[t]{3}{*}{ España } & $\begin{array}{l}\text { Observatorio de lgualdad de Género de la Universidad } \\
\text { Rey Juan Carlos }\end{array}$ & http://observatorio.inie.ucr.ac.cr/ \\
\hline & $\begin{array}{c}\text { Observatorio de Políticas Públicas en Materia de } \\
\text { Discapacidad, Atención a la Diversidad e lgualdad de } \\
\text { oportunidades }\end{array}$ & http://www.ce10udc.com/ \\
\hline & Observatorio Estatal de Violencia de Género & $\begin{array}{l}\text { http://www.msssi.gob.es/ssi/violenciaGenero/ObservatorioEs- } \\
\text { tatal/home.htm }\end{array}$ \\
\hline
\end{tabular}

Fuente: Gutiérrez y Vásquez (2017) 


\section{OBSERVATORIOS DE POLÍTICAS PÚBLICAS A PARTIR DE ALIANZAS PRIVADAS O PÚBLICO-PRIVADAS}

Existen una serie de observatorios que se forman a partir de alianzas público- privadas entre diferentes actores como universidades, empresas privadas y organizaciones no gubernamentales. También, existen una serie de observatorios organizados en red.

Los organismos de violencia social de género de México, como red, están conformados a partir de observatorios. Cada uno cuenta con un comité técnico local integrado por un grupo de especialistas que coordinan las actividades y captan información a través una red de información. A su vez, esta red dispone de dos estructuras: la Coordinación Sectorial de Instituciones, que está constituida por entidades públicas, privadas y de la sociedad civil como son centros de información, universidades, entre otros que ya poseen algún tipo de información.

Por su parte, la Red Territorial de Unidades de Información y Monitoreo es responsable del acopio y registro de la información, entre ellas, las denuncias en cuestiones de violencia social y de género. A su vez, en el ámbito nacional, se tiene el Grupo Interdisciplinario de Investigación e Intervención Social (GIIIS) como un cuerpo multi- profesional vinculados al sector público, privado y de la sociedad civil. El GIIS se encarga de analizar las condiciones sociales que repercuten en la violencia contra la mujer y las de género; por consiguiente, produce diversos estudios, investigaciones, artículos, entre otros sobre el tema. Este grupo es de carácter abierto y voluntario.

El observatorio de políticas públicas de Medellín, en Colombia, fundamenta su operación en la red de dependencias gubernamentales, organizaciones y actores sociales, las cuales interactúan continuamente alrededor de temas prioritarios de ciudad, suministran y apoyan el análisis de información necesaria para la producción de conocimiento. Para tal efecto, conforman mesas de trabajo interorganizacionales, estandarizan procesos de generación de información y desarrollan actividades con el propósito de lograr una mayor eficacia de las políticas públicas.

\section{OBSERVATORIOS DE POLÍTICAS PÚBLICAS POR MATERIA}

Los observatorios de políticas públicas muchas veces se constituyen para dar seguimiento a unas políticas específicas en diversos ámbitos, como: pequeñas y medianas empresas (pymes), control de trabajo, ambiente, género, familia, trabajo, entre otros, como puede observarse en la tabla 4.

TABLA 4

OBSERVATORIOS DE POLÍTICAS PÚBLICAS EN UN ÁMBITO ESPECÍFICO

\begin{tabular}{|c|c|c|}
\hline País & Observatorio & Ámbito \\
\hline Argentina & Observatorio pyme & Pymes \\
\hline Argentina & Observatorio Argentina de Droga & Drogas \\
\hline Brasil & Observatorio de Política Nacional de Control de Tabaco & Control de Tabaco \\
\hline España & Observatorio de Igualdad de Género de la Universidad Rey Juan Carlos & Género \\
\hline España & Observatorio de Políticas de Agua & Agua \\
\hline Paraguay & Observatorio Laboral & Trabajo \\
\hline
\end{tabular}

Fuente: Gutiérrez y Vásquez (2017) 
En el caso del Observatorio de Chilecompra, se encuentra dedicado a la política pública de compras públicas de Chile. Para tal efecto, despliega una serie de actividades relacionadas con la revisión diaria de los procesos de compra publicados por las organizaciones públicas de Chile. En sus diferentes etapas, emite recomendaciones a esas organizaciones para mejorar los procesos de compra y mantiene comunicación con los diferentes ciudadanos sobre la temática de las compras públicas.

\section{CONCLUSIONES}

En los primeros años del siglo XXI ha existido una proliferación de los observatorios de políticas públicas, cuya fundación tiene su origen en distintas organizaciones. Aparecen como producto de la necesidad de los gobernantes y de la Sociedad Civil en general, de conocer la interacción de los diferentes actores públicos y privados, en la formulación de las políticas públicas.

La nueva gobernanza, impregnada de la gobernabilidad, exige participación ciudadana en las políticas públicas que cada vez son más complejas debido a la heterogeneidad de los mismos actores, a la interdependencia e interconexión local e internacional y con acceso absoluto a la información. Los observatorios vienen a llenar esa necesidad de la nueva gobernanza de conocer de las políticas públicas.

Se reconoce que la eficacia y eficiencia de las políticas públicas dependerá, en sus diferentes etapas, de una amplia participación de los actores provenientes del Estado, de la sociedad civil y del mercado; el Estado es un actor importante, pero no determinante de la política pública. El anterior reconocimiento se origina en un marco de reivindicación y de ampliación de los derechos ciudadanos, que ha hecho posible el gobierno abierto. Con ello, brinda un paso más en el perfeccionamiento de la democracia representativa.
Los observatorios en políticas públicas son entes que, en forma constante y permanente, sin interrupción alguna, son observadores del desarrollo de las políticas públicas. Por lo tanto, a todos los observatorios les es común la investigación en políticas públicas $y$, por ende, son fuentes significativas de información, de capacitación y de asesoría en el mejoramiento de las políticas públicas regionales, nacionales y locales.

Del acervo de conocimiento producido por los observatorios de políticas públicas, se nutren las nuevas capacidades de los gestores públicos, las cuales se basan en el reconocimiento de que hoy en día la ciudadanía se ha reivindicado y ampliado junto con la sociedad civil. Por ello, la eficiencia y la eficacia de las políticas públicas dependerán de un conglomerado de actores, públicos y privados (estos últimos provenientes de la sociedad civil y del mercado) y del reconocimiento de la importancia de las instituciones, formales e informales. Esto último ha sido un llamado insistente desde el ámbito de los teóricos neoinstitucionales.

La fiscalización y el monitoreo de la formulación y de la implementación de las políticas públicas -en particular, sobre el eje de la transparencia y la utilización eficiente de los fondos públicosson aspectos que se encuentran con mayor intensidad en los observatorios provenientes de la sociedad civil, de conformidad con las nuevas características de la ciudadanía ya mencionadas.

Esta mayor vigilancia de los asuntos públicos de los observatorios de la sociedad civil se hace evidente desde los signos externos que los identifican, el signo que se utiliza con frecuencia es un "ojo", como señala de vigilancia o monitoreo de lo que ocurre. Los observatorios asociados a los entes públicos cuentan con una agenda concordante con las prioridades y con las exigencias de los gobernantes del turno, lo que no necesariamente coincide con los observatorios prove- 
nientes de la académica, que tienen su propia agenda, por naturaleza independiente.

En el caso de Costa Rica, no es comprensible que se carezca de un observatorio de políticas públicas general, pues el país tiene una larga tradición democrática, donde la ciudadanía ha sido reivindicada y ampliada en las últimas décadas. Solo cuenta con unos pocos observatorios en la academia, cuyos objetivos no necesariamente se relacionan con el estudio de una política pública específica, sino que su estudio es, en cierta forma, colateral.

\section{REFERENCIAS}

Breppe, P., \& Pereyra, A. (2014). Ciudadanía en las políticas públicas de América Latina. Revista Margen $75,1-7$.

Instituto Nacional de Administración Pública (INAP). (2014). La función social de los observatorios, el caso del Observatorio Latinoamericano de la Administración Pública. México, D.F., México: INNO, Serie Praxis.
Mayorga, F., \& Córdoba, E. (2007). Gobernabilidad y gobernanza en América Latina. Ginebra, Suiza: Instituto de Investigación y Debate sobre la Gobernanza.

Natal., A., \& Díaz, O. (2014). ¿Qué son y cómo funcionan los Observatorios Ciudadanos? México, D.F., México: Ediciones Gernika, S. A. Universidad Autónoma Metropolitana.

Scharpf, F. (1997). Games Real Actors Play: Actor-centered institutionalism in Policy Research. New York, United States of America: Routledge Taylor \& Francis Group.

Subirats, J., Knoepfel, P., Corinne L., \& Varones, F. (2008). Análisis y gestión de políticas públicas. Barcelona, España: Editorial Ariel.

Vásquez, J. A. (2014). La institucionalidad de la Calidad de los Servicios Públicos en Costa Rica. Revista Nacional de Administración, 5(1), 31-45.

Zurbriggen, C. (2008). El institucionalismo centrado en los actores: Una perspectiva analítica en el estudio de las políticas públicas. Revista de Ciencia Política (Santiago), 26(1), 67-83.

Recibido:14 de febrero de 2018 Aceptado:10 de septiembre de 2018 